\title{
Sinonasal symptom-related sleep disorders before and after surgery for nasal polyposis*
}

\author{
Duc Trung Nguyen', Fabien Arous², Patrice Gallet', Marylisa Felix-Ravelo', \\ Phi-Linh Nguyen-Thi ${ }^{3}$, Cécile Rumeau ${ }^{1,2}$, Roger Jankowski ${ }^{1,2}$ \\ ' University hospital of Nancy, Department of Otorhinolaryngology - Head and Neck Surgery, Nancy, France \\ 2 Faculty of medicine, University of Lorraine, France \\ ${ }^{3}$ University hospital of Nancy, Plateforme d'Aide à la Recherche Clinique - PARC. Unité ESPRI - BioBase. Méthodologie - \\ Règlementation - Biostatistique, Nancy, France
}

Rhinology 55: 262-268, 2017

https:://doi.org/10.4193/Rhino16.016

*Received for publication:

January 17, 2016

Accepted: December 5, 2016

\begin{abstract}
Background: Patients with nasal polyposis (NP) complain of several sinonasal symptoms that impact their sleep and quality of life. However, data on sleep disorders related to NP symptoms, before and after surgery, is poor. The aim of the present study was to analyze sleep complaints related to each NP symptom, before and after surgery, using the Dynachron questionnaire.

Methodology: 63 patients operated for NP were included in this prospective study. They filled the DyNaChron questionnaire one day before surgery (V0), 6 weeks (V1) and 7 months (V2) after surgery. The self-ratings (0-10 point visual analog scale) of nasal obstruction, anterior rhinorrhea, postnasal discharge, cough and 5 items related to sleep disturbances, due to each symptom of chronic nasal dysfunction, were extracted from the questionnaire and analyzed.
\end{abstract}

Results: There was significant improvement of symptoms and symptom-related sleep disturbance scores at V1 and V2 compared to baseline scores. Before surgery, moderate/severe sleep disorders that patients attributed to nasal obstruction (the patient thinks it is due to nasal obstruction rather than a clinical test to show nasal obstruction) or anterior rhinorrhea were reported in two thirds of patients, postnasal discharge in one half, and chronic cough in one third. After surgery, less than $10 \%$ of patients reported moderate/severe sleep disorders at $\mathrm{V} 1$. There was a mild increase of patients who rated moderate/severe sleep disorders at V2 in comparison to V1. The correlation between scores of nasal obstruction and its impacts on sleep quality was weak before surgery and strong afterwards.

Conclusion: Nasalization improved sleep quality significantly at 6 weeks and at 7 months after surgery. However, there was a mild increase of complaints related to postnasal discharge and cough at 7 months after surgery.

Key words: nasal polyposis, sleep, Dynachron questionnaire, nasal obstruction, rhinorrhea, postnasal discharge

\section{Introduction}

Nasal polyposis (NP) is a chronic inflammatory disease, specific to the ethmoidal mucosa according to evo-devo arguments ${ }^{(1)}$. A high prevalence of pathological sleep dysfunction related to this disease was reported compared to the general population ${ }^{(2)}$. $72 \%$ to $75 \%$ of patients with chronic rhinosinusitis (CRS) reported abnormal quality of sleep ${ }^{(3,4)}$. Furthermore, patients with NP usually reported staggering effects on daily performance and quality of life (QOL) due to sleep disturbances ${ }^{(2)}$.

Mechanisms of sleep dysfunction in CRS, especially in NP are not yet clear. The aetiology of sleep disturbances is multifactorial. It was suggested that pro-inflammatory cytokines, hormones, and bacterial cell wall products influence the structural sleeping patterns (such as IL-I $\beta$, tumor necrosis factor- $\alpha$ (TNF- $\alpha$ ), and growth hormone releasing hormone (GHRH) for non rapid eye movement sleep, and Nitric Oxide and prolactin for regulating rapid eye movement sleep ${ }^{(5)}$. On the other hand, nasal obstruction plays an important role in sleep-disordered breathing. Poor quality of sleep was observed when artificially inducing occlusion of the nose by significantly increasing the number of 
apneas, hypopneas, sleep arousals and awakenings, and loss of deep sleep ${ }^{(6,7)}$. Moreover, other symptoms such as sneezing, rhinorrhea and nasal pruritus may also contribute to worsen the sleep quality in patients with allergic rhinitis ${ }^{(8)}$.

Patients with NP complain of several sinonasal symptoms that impact their sleep quality and quality of life, especially when NP is recalcitrant to medical treatment ${ }^{(9)}$. The improvement of sleep quality after endoscopic sinus surgery was reported in patients with CRS ${ }^{(3,4)}$ and in a series of 27 patients with NP ${ }^{(10)}$. However, data on sleep disorders related to each symptom of NP before and after surgery is poor. The knowledge of symptom-related sleep disturbances may contribute to a better understanding of the mechanisms of sleep disorders in NP. The aim of the present study is to analyze sleep complaints related to each symptom before and after surgery for NP using the Dynachron questionnaire.

\section{Patients and methods}

\section{Patients}

Sixty-three consecutive patients that underwent endoscopic surgery for NP at our tertiary care hospital were included in this prospective study. All patients filled out the DyNaChron questionnaire (Dysfonctionnement Nasal Chronique in French or Chronic Nasal Dysfunction in English) ${ }^{(11)}$ one day prior to surgery (V0), 6 weeks (V1) and 7 months (V2) after surgery. Nasal polyposis was diagnosed based on the presence of polyps in both nasal cavities by endoscopic examination during the initial visit. The staging of polyp grade was given according to Malm's classification ${ }^{(12)}$. Surgery was suggested when medical treatment failed to control the symptoms. All patients were operated on both ethmoids, according to the nasalization procedure (or radical ethmoidectomy procedure) ${ }^{(13)}$, with the middle turbinates being preserved whenever possible. The aim of the nasalization procedure was to remove all bony lamellas and mucosa inside the ethmoidal labyrinths as well as the mucosa on the lamina papyracea, skull base, lateral side of the middle/superior turbinates and conchal lamina. The procedure begins by a dissection of the ethmoidal labyrinth between middle turbinate and medial orbital wall. Then, the middle turbinate can easily be lateralized in order to operate in the olfactory cleft in which the superior and supreme meatus, as well as the sphenoethmoidal recess and sphenoidal ostium can be addressed. The middle turbinates were removed in case of polyps stemming from the mucosa of medial side of the middle turbinate because of high risk of polyp recurrence. The mucosa inside the ethmoidal labyrinth and of its walls (orbital wall, ethmoidal roof and lateral side of the ethmoidal turbinate wall) is removed as completely as possible. The mucosa of the olfactory cleft is preserved as much as possible. Ostia of the maxillary, frontal and sphenoidal sinuses are liberated, but not broadened if not necessary in order to preserve the physiology of the paranasal sinus cavities. Septoplasty was performed when necessary. No systemic corticosteroid or antibiotic treatment was systematically given before or after surgery. Local care was started the day after surgery, consisting of nasal washing at least three times a day with an isotonic saline solution using a syringe, and topical steroids once daily. These topical treatments were also strongly recommended for longterm usage. This study was approved by the Institutional Review Board of the University Hospital of Nancy, France.

\section{Evaluation of symptoms}

All data was collected prospectively using the DyNaChron questionnaire, which has been validated on a large sample of patients in a prospective multicenter study ${ }^{(11)}$. The self-ratings of nasal obstruction, anterior rhinorrhea, postnasal discharge, cough and 5 items related to sleep disturbances, due to each symptom of chronic nasal dysfunction, were extracted from the questionnaire and analyzed. The 5 sleep-related items were: difficulty falling asleep due to nasal obstruction (item 6), waking up at night due to nasal obstruction (arousals related to nasal obstruction) (item 7), waking up at night due to an excessively moist or runny nose (arousals related to anterior rhinorrhea) (item 20), sleep disorders (difficulty falling asleep or waking up at night) due to postnasal discharge (item 32), and sleep disorders due to cough (item 68). Each complaint was scored using the 0-10 VAS grading scale, with 0 meaning no discomfort at all and 10 meaning unbearable discomfort. Patients were asked to rate their symptoms over the last 15 days.

\section{Statistical analysis}

Continuous variables were expressed as mean \pm standard deviation and categorical variables were expressed as frequencies and percentages. Sleep quality was rated and grouped in categories of none (0), mild (1-3), moderate (4-6), and severe (7-10) to express the intensity of sleep disorders. All data was examined for normality using Shapiro-Wilk's tests. Chi-square or, when necessary, Fisher's exact tests were used for categorical variables. For continuous variables, because of the non-normal distribution of samples, the Wilcoxon-Mann-Whitney test was used to compare the VAS scores of each post-op evaluation to baseline scores as well as scores from V1 versus V2. The KruskalWallis test was used to compare three polyp-staging subgroups. The Spearman's correlation test was performed to examine any relationship between the symptoms' scores and symptom-related sleep disturbances' scores. Statistical analyses were performed by means of the SAS v9.1 software (SAS Inst., Cary, NC, USA) where the two-sided $p$-value of less than 0.05 was significant.

\section{Results}

A total of 63 patients ( 32 males and 31 females; mean age of 51 \pm 12.8 years) filled the DyNaChron questionnaire at $\mathrm{V} 0, \mathrm{~V} 1$ (mean days, $40.9 \pm 9.7$ ) and V2 (mean days, 261.7 \pm 29.8 ). Thirty-seven 
Table 1. Mean self-rating scores of each symptom and its impact on sleep quality (VAS) before and after surgery (Wilcoxon- Mann- Whitney test).

\begin{tabular}{|c|c|c|c|c|c|c|}
\hline & Vo & V1 & V/2 & $\begin{array}{l}\text { p-value } \\
\text { V1 vs V0 }\end{array}$ & $\begin{array}{l}\text { p-value } \\
\text { V6 vs V0 }\end{array}$ & $\begin{array}{l}\text { p-value } \\
\text { V6 vs V1 }\end{array}$ \\
\hline Nasal obstruction & $6.76 \pm 2.24$ & $1.59 \pm 1.94$ & $1.60 \pm 2.23$ & $<0.0001$ & $<0.0001$ & 0.91 \\
\hline $\begin{array}{l}\text { Difficulty falling asleep due to nasal } \\
\text { obstruction }\end{array}$ & $5.40 \pm 2.94$ & $0.78 \pm 1.56$ & $0.95 \pm 1.75$ & $<0.0001$ & $<0.0001$ & 0.46 \\
\hline Arousals related to nasal obstruction & $4.95 \pm 2.92$ & $0.78 \pm 1.48$ & $0.79 \pm 1.77$ & $<0.0001$ & $<0.0001$ & 0.99 \\
\hline Anterior rhinorrhea & $7.10 \pm 2.39$ & $2.87 \pm 2.48$ & $2.87 \pm 2.62$ & $<0.0001$ & $<0.0001$ & 1.0 \\
\hline $\begin{array}{l}\text { Arousals related to anterior rhinor- } \\
\text { rhea }\end{array}$ & $4.51 \pm 3.09$ & $0.78 \pm 1.58$ & $1.03 \pm 1.67$ & $<0.0001$ & $<0.0001$ & 0.29 \\
\hline Postnasal discharge & $5.08 \pm 3.25$ & $1.78 \pm 2.19$ & $2.79 \pm 2.75$ & $<0.0001$ & $<0.0001$ & 0.002 \\
\hline $\begin{array}{l}\text { Sleep disorders related to postnasal } \\
\text { discharge }\end{array}$ & $3.87 \pm 3.26$ & $0.70 \pm 1.58$ & $1.21 \pm 2.03$ & $<0.0001$ & $<0.0001$ & 0.08 \\
\hline Cough & $3.11 \pm 3.24$ & $1.21 \pm 2.18$ & $1.68 \pm 2.58$ & $<0.0001$ & 0.0003 & 0.076 \\
\hline Sleep disorders related to cough & $2.63 \pm 3.12$ & $0.79 \pm 1.94$ & $0.98 \pm 2.17$ & $<0.0001$ & $<0.0001$ & 0.38 \\
\hline
\end{tabular}

Table 2. Correlations between symptom scores and their impact on sleep quality before and after surgery (Spearman's correlation).

\begin{tabular}{|c|c|c|c|}
\hline r63 (p-value) & Vo & V1 & $\mathbf{V} \mathbf{2}$ \\
\hline Nasal obstruction and Difficulty falling asleep due to nasal obstruction & $0.3(p=0.016)$ & $0.71(p<0.0001)$ & $0.78(p<0.0001)$ \\
\hline Nasal obstruction and Arousals related to nasal obstruction & $0.2(p=0.11)$ & $0.75(p<0.0001)$ & $0.68(p<0.0001)$ \\
\hline Anterior rhinorrhea and Arousals related to anterior rhinorrhea & $0.49(p<0.0001)$ & $0.54(p<0.0001)$ & $0.64(p<0.0001)$ \\
\hline Postnasal discharge and Sleep disorders related to postnasal discharge & $0.58(p<0.0001)$ & $0.54(p<0.0001)$ & $0.69(p<0.0001)$ \\
\hline Cough and Sleep disorders related to cough & $0.82(p<0.0001)$ & $0.79(p<0.0001)$ & $0.82(p<0.0001)$ \\
\hline
\end{tabular}

patients (58.7\%) had a history of previous sinonasal surgery for NP (polypectomy or functional ethmoidectomy or nasalisation). Polyps were given a stage-grade of 1 in 8 patients (12.7\%), grade 2 in 20 (31.8\%) and grade 3 in 35 (55.6\%). Middle turbinates could be preserved in 31 patients (49.2\%).

\section{Self-rating of nasal symptoms and symptom-related sleep disturbances}

Table 1 shows mean self-rating scores of each symptom and its impact on sleep quality before and after surgery. There was a significant improvement of symptoms and symptom-related sleep disturbance scores at V1 and V2 compared to baseline scores $(p<0.0001)$. These scores were not significantly different between $\mathrm{V} 1$ and $\mathrm{V} 2$, except the score of postnasal discharge, which increased at V2 compared to that at V1 $(p=0.002)$. Consequently, the score of sleep disorders related to this symptom tended to increase at V2 $(p=0.08)$. The score of cough tended to be higher at V2 than V1 ( $p=0.076)$ but there was no significant difference of sleep disorder score related to this symptom between $\mathrm{V} 1$ and $\mathrm{V} 2(\mathrm{p}=0.38)$.

Correlations between symptom scores and their impact on sleep quality before and after surgery are shown in Table 2. Before surgery, there was a weak correlation between scores of nasal obstruction and impact on sleep quality ( $r 63=0.3$ for difficulty falling asleep and 0.2 for waking up at night). In other words, some patients rated a high score for nasal obstruction but low score for difficulty falling asleep and waking up at night and vice versa. In contrast, this correlation was strong at V1 and V2 ( $r 63$ between 0.68 and $0.78, p<0.0001$ ). The correlations between (anterior and posterior) rhinorrhea and its impact on sleep were moderate at V0 and V1 but strong at V2. The self-rating score of cough was strongly correlated to the score of sleep disorder caused by this symptom at all three assessments $(p=0.82,0.79$ and 0.82 respectively, $\mathrm{p}<0.0001$ ).

\section{Intensity of sleep disorders before and after surgery}

Figures 1-3 shows the intensity of sleep disorders according each symptom at V0 (Figure 1), V1 (Figure 2) and V2 (Figure 3). Before surgery, moderate/severe sleep disorders were reported by about two-thirds of patients for nasal obstruction and anterior rhinorrhea, by half for postnasal discharge, and by one-third for cough. At 6 weeks and 7 months after surgery, there was a remarkable improvement of sleep quality. No sleep disorder was 
reported by about $70 \%$ of patients. Less than $10 \%$ of patients reported moderate/severe sleep disorders at V1. However, there was a slight increase of patients that rated moderate/severe sleep disorders at V2 in comparison to V1, especially linked to postnasal discharge and nasal obstruction.

\section{Symptom-related sleep disorders according to polyp sta- ging}

Table 3 shows the mean scores of sleep quality related to each symptom according to polyp staging. Before surgery, patients with grade 3 polyps reported more sleep discomfort, even if not significant, due to nasal obstruction and anterior rhinorrhea, than those with other grades. By contrast, patients with grade 1 polyps complained of more important sleep disorders due to postnasal discharge than other grades $(p=0.03)$. After surgery, there was no significant difference of symptom-related sleep disorders between grade groups.

Symptom-related sleep disorders according to middle turbinate status

There was no statistical difference of mean scores of sleep quality related to each symptom between patients with middle turbinate preservation and those with middle turbinate resection at V1 and V2 ( $p>0.05)$.

\section{Discussion}

Our findings show that endoscopic surgery for NP improves sleep quality at 6 weeks and at 7 months after surgery due to the improvement of nasal symptoms, assessed by the Dynachron questionnaire. There was a mild tendency of re-apparition of some symptoms at 7 months after surgery, such as postnasal discharge and cough, leading to a parallel mild increase of sleep disorders at this point of assessment. Both data suggest a pathophysiological link between nasal dysfunction and sleep disorders.

Alt et al. ${ }^{(4)}$ reported a reduction of the prevalence of poor sleep from $72.1 \%$ before surgery to $56.6 \%$ after surgery for CRS, using the Pittsburgh Sleep Quality Index (PSQI) and the 22-item SinoNasal Outcome Test (SNOT-22). However, the authors included patients with CRS without distinguishing NP from CRSsNP. In addition, different surgical procedures were performed in their series (uni- or bilateral maxillary antrostomy, partial or total ethmoidectomies). In contrast, our population was more homogenous, including only patients with NP who underwent the same surgical procedure, obtaining more specific results for a well-defined population.

Sleep disorders associated to NP consist of difficulty in falling asleep, difficulties in falling asleep after nocturnal awakening, involuntary inadequate sleep time, inefficient sleep, frequent

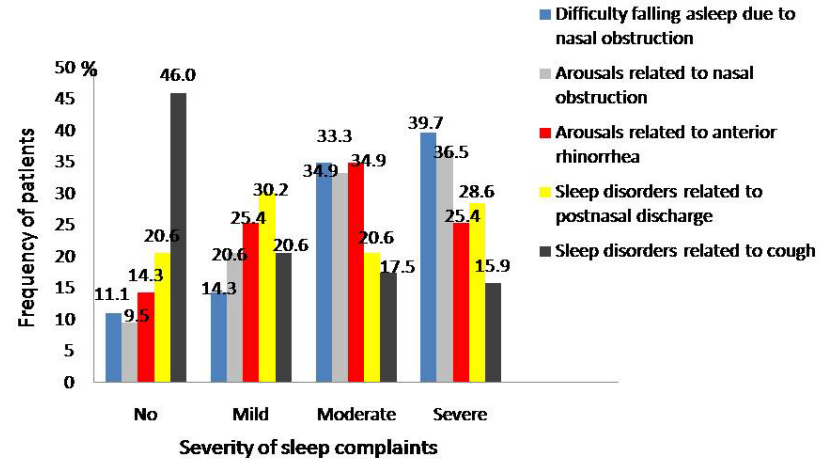

Figure 1. Frequency of patients in each group according to the intensity of symptom-related sleep disorders before surgery (V0).

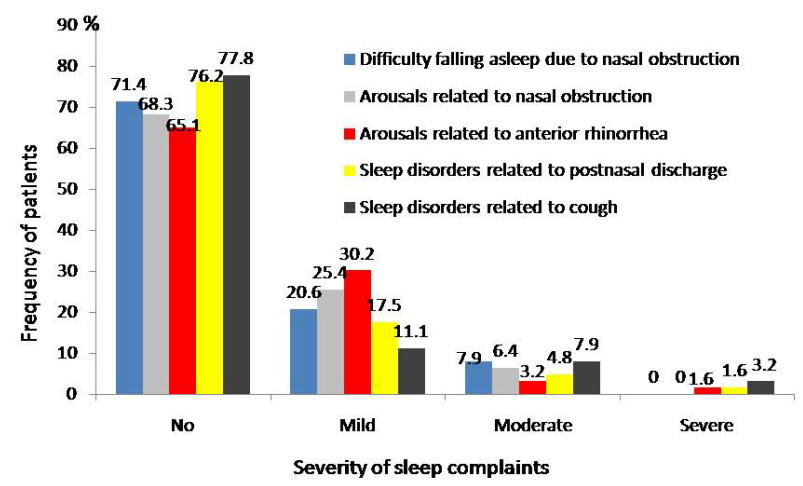

Figure 2. Frequency of patients in each group according to the intensity of symptom-related sleep disorders at 6 weeks after surgery (V1).

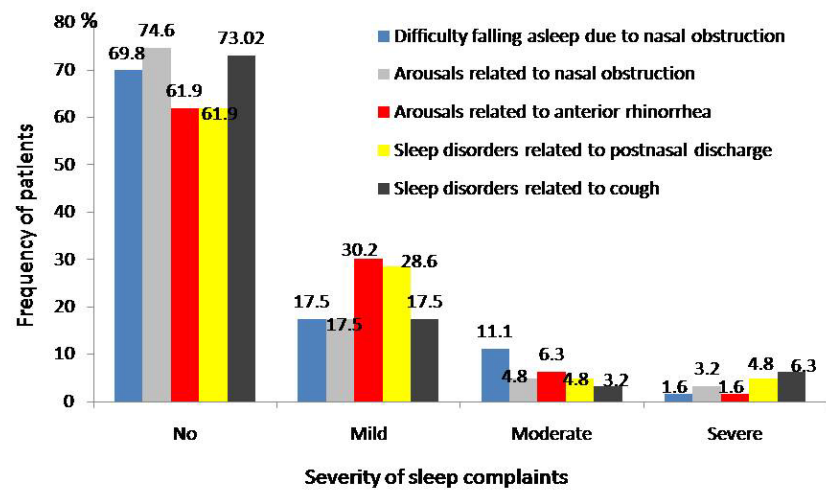

Figure 3. Frequency of patients in each group according to the intensity of symptom-related sleep disorders at 7 months after surgery (V2).

arousals leading to sleep fragmentation, and early awakening

(2). The mechanism of sleep disorders in patients with NP seems multifactorial ${ }^{(5)}$. It may be due to mechanical blockage by the polyps and reduced cross-section area by nasal inflammatory mucosa leading to the increase of the upper airway resistance, and/or to the systemic diffusion of pro-inflammatory cytokines. The assessment of sleep disorders related to each symptom using the Dynachron questionnaire allows to evaluate complaints caused by mechanical blockages (nasal obstruction) 
Table 3. Mean scores of sleep quality related to each symptom according to polyp staging (8 grade 1, 20 grade 2; and 35 grade 3) (Kruskal-Wallis test).

\begin{tabular}{|c|c|c|c|c|c|c|c|c|c|c|c|c|}
\hline & & $\begin{array}{r}\text { VO } \\
\text { Polyp sta }\end{array}$ & jing & & & $\begin{array}{r}\text { V1 } \\
\text { Polyp st: }\end{array}$ & ing & & & $\begin{array}{r}\text { V/2 } \\
\text { Polyp sta }\end{array}$ & jing & \\
\hline & 1 & 2 & 3 & $\mathbf{p}$ & 1 & 2 & 3 & $\mathbf{p}$ & 1 & 2 & 3 & $\mathbf{p}$ \\
\hline $\begin{array}{l}\text { Difficulty } \\
\text { falling asleep } \\
\text { due to nasal } \\
\text { obstruction }\end{array}$ & $4.38 \pm 3.50$ & $4.65 \pm 3.20$ & $6.06 \pm 2.54$ & 0.16 & $0.75 \pm 1.39$ & $0.95 \pm 1.64$ & $0.69 \pm 1.59$ & 0.49 & $1.00 \pm 2.83$ & $1.05 \pm 1.50$ & $0.89 \pm 1.64$ & 0.37 \\
\hline $\begin{array}{l}\text { Arousals } \\
\text { related to nasal } \\
\text { obstruction }\end{array}$ & $4.00 \pm 3.51$ & $4.25 \pm 2.95$ & $5.57 \pm 2.68$ & 0.14 & $0.63 \pm 1.19$ & $1.00 \pm 1.65$ & $0.69 \pm 1.45$ & 0.64 & $1.13 \pm 3.18$ & $0.75 \pm 1.07$ & $0.74 \pm 1.72$ & 0.37 \\
\hline $\begin{array}{l}\text { Arousals rela- } \\
\text { ted to anterior } \\
\text { rhinorrhea }\end{array}$ & $3.25 \pm 3.06$ & $4.05 \pm 3.43$ & $5.06 \pm 2.85$ & 0.23 & $0.63 \pm 1.19$ & $0.85 \pm 1.39$ & $0.77 \pm 1.78$ & 0.90 & $2.13 \pm 3.04$ & $0.75 \pm 1.02$ & $0.94 \pm 1.51$ & 0.80 \\
\hline $\begin{array}{l}\text { Sleep disorders } \\
\text { related to post- } \\
\text { nasal discharge }\end{array}$ & $5.25 \pm 3.81$ & $2.35 \pm 2.58$ & $4.43 \pm 3.25$ & 0.03 & $2.13 \pm 2.95$ & $0.50 \pm 1.28$ & $0.49 \pm 1.15$ & 0.12 & $2.50 \pm 3.25$ & $0.95 \pm 1.50$ & $1.06 \pm 1.89$ & 0.29 \\
\hline $\begin{array}{l}\text { Sleep disorders } \\
\text { related to } \\
\text { cough }\end{array}$ & $2.00 \pm 3.42$ & $2.65 \pm 3.00$ & $2.77 \pm 3.19$ & 0.83 & $1.63 \pm 3.46$ & $0.45 \pm 1.19$ & $0.80 \pm 1.83$ & 0.54 & $1.13 \pm 3.18$ & $0.70 \pm 1.42$ & $1.11 \pm 2.31$ & 0.66 \\
\hline
\end{tabular}

and nasal inflammation (rhinorrhea, cough), whereas other questionnaires do not allow to distinguish the exact symptoms that contribute to sleep disorders. Moreover, the assessment of residual symptoms during the follow-up after surgery may provide more accurate information on the first signs of recurrence of the disease ${ }^{(14)}$.

The majority of studies focus on nasal obstruction in sleep disorders ${ }^{(4,15)}$ but few focus on other symptoms such as anterior rhinorrhea, postnasal discharge, chronic cough, and/or pain. Nasal obstruction has been identified as an independent risk factor in the pathogenesis of sleep disordered breathing by increasing the nasal airway resistance leading to an increase of the pharyngeal airway's negative pressure and the its collapsibility ${ }^{(16)}$. This can worsen obstructive sleep apnea (OSA) and snoring. Behind the blockage due to polyps, the inflammatory processes of CRS may increase the upper airway's resistance by reducing the nasal cross-sectional area (17). In parallel, in the improvement of nasal permeability by polyp removal, the nasalization may help to control the inflammation of the upper airway by removing, as much as possible, the diseased mucosa in the ethmoids. In consequence, the upper airway's resistance and the production of pro-inflammatory cytokines may decrease. Recently, Jiang et al explored the relationship of CRS and sleep-disordered breathing by using the SNOT-20, Epworth Sleepiness Scale (EpSS), and polysomnography among 139 patients, which included 70 patients with NP ${ }^{(18)}$. The authors found that sleep problems were correlated with the symptom of nasal obstruction but nasal polyps did not seem to worsen sleep problems. However, the authors assessed sleep problems using the EpSS that only evaluates daytime sleepiness, which can have many causes. The EpSS is not a good tool to evaluate sleep problems because of its low sensitivity (0.46) and specificity (0.60) in the identification of OSA ${ }^{(19)}$. Moreover, the grades of polyps were not detailed in this paper.

Behind the mechanical blockage, the inflammatory phenomenon may play an important role in sleep disorders of patients with CRS, especially in patients with NP. Karosi et al. ${ }^{(20)}$ showed that patients with NP had increased expression of TNF-a receptors. Lennard et al demonstrated higher mucosal levels of IL-1 $\beta$, IL-6, IL-8, TNF-a in patients with CRS than in controls ${ }^{(21)}$. It has been shown that a lower dose of IL-1 $\beta$ increases sleep whereas a high dose inhibits sleep ${ }^{(22)}$. Rotenberg et Pang ${ }^{(23)}$ reported that functional endoscopic sinus surgery improved sleep outcomes (using EpSS, PSQI and SNOT-22) but not nasal obstruction (using Nasal Obstruction Symptom Evaluation score) at 6 months among 53 patients with CRSsNP (patients with NP were excluded). Our findings show that endoscopic surgery improved significantly the symptoms reflecting local inflammation such as nasal obstruction, anterior rhinorrhea, postnasal discharge, and chronic cough in patients with NP. Hence, sleep problems related to these symptoms, local inflammation to be precise, improved during the postoperative follow-up. Interestingly, sleep problems tended to mildly go up at 7 months after surgery, and were linked to the increase of postnasal discharge and chronic cough, which are symptoms of local inflammation of the sinonasal mucosa.

The weak correlation between nasal obstruction and sleep dis- 
orders linked to this symptom before surgery may be due to the habituation of patients with chronic nasal obstruction. However, strong correlations between nasal obstruction and sleep disorders linked to this symptom were observed after surgery. Thus, they can accurately attribute this symptom to sleep disorders that appear after surgery. However, we have no answer to why patients are able to attribute nasal obstruction as a cause of sleep difficulties post-surgery but not beforehand. Therefore, further studies are necessary. On the other hand, for anterior and posterior rhinorrhea, patients seem able to attribute these symptoms as causes of sleep disorders, both, before and after surgery. In contrast, the link between cough and its impact on sleep quality was very strong before and after surgery, perhaps because this symptom is an irresistible reflex, which by its self disturbs sleep.

Theoretically, the higher the polyp grade, the higher the severity of nasal obstruction. Consequently, the upper airway's resistance should increase according to the severity of polyp grades. Thus, the self-rating scores should be higher in patients with advanced polyp grades. Actually, we failed to demonstrate a difference between groups, though the self-rating scores were higher in patients with grade 3 polyps. This may be due to the small number of subjects in each group. Regarding postnasal discharge, patients with grade 1 polyp had worse scores than those with grade 2 and 3 . This may be due to the indication bias or the different presentations of the disease. In other words, surgery was recommended to patients with small polyps because the inflammation was not controlled with medical treatment leading to patients' discomfort. After surgery, there was no difference between groups as polyps were removed and the inflammation was better controlled by the removal of the mucosa inside of the ethmoidal labyrinths as much as possible complete (13). Some patients in our study underwent surgery even after experiencing no/mild severity sleep disturbances (Figure 1). Among these patients, the impact of the symptoms, according to the Dynachron questionnaire, were more significant on their quality of life and/or professional activities rather than their sleeping patterns. Surgery was suggested to our patients only when medical treatment failed to control the symptoms. Our previous study showed that $98.46 \%$ of patients operated for NP reported at least 3 symptoms before surgery ${ }^{(14)}$.

Our study allows to investigate sleep disordered breathing specifically in patients with NP before and after surgery. Moreover, we try to analyze sleep disorders related to each one of these patients' complaints to better understand the relationship between chronic nasal dysfunction and sleep disorders. However, our study did not include a combination of the Dynachron questionnaire with more general questionnaires, which may have created limitations for patients to accurately attribute each symptom as a cause of sleep disorders. Perhaps, further studies on patients with only one single symptom could be interesting and easier to assess. In the other hand, the present study was performed without objective airway function measurements neither any objective quantification of the symptom severity. However, there are no data available at the moment in the quantification of the symptom severity in patients suffering from chronic (sino)nasal inflammation ${ }^{(24)}$.

\section{Conclusion}

Nasalization improved sleep quality significantly at 6 weeks and at 7 months after surgery. However, there was a mild increase of complaints related to postnasal discharge and cough at 7 months after surgery. The Dynachron questionnaire may contribute to the analysis of sleep quality in relation to each symptom of NP before and after surgery. It helps clinicians to personalize the treatment when possible according to the persistence of either particular symptom follow-up visits. The knowledge on which symptom is related to sleep disturbances may contribute to a better understanding of sleep disorders in patients with NP.

\section{Authorship contribution}

DTN: study design, data collection, writing the paper; DTN, P-L $\mathrm{N}-\mathrm{T}$ : data analysis, FA: manuscript corrections; PG,MFR, CR: discussion; RJ: discussion, study overview

\section{Conflict of interest}

No financial disclosures; conflicts of interest: none

\section{References}

1. Jankowski R. The Evo-Devo Origin of the Nose, Anterior Skull Base and Midface. 1st edition. Paris, France: Springer-Verlag, 2013 ISBN 978-2817804217.

2. Serrano E, Neukirch F, Pribil C, et al. Nasal polyposis in France: impact on sleep and quality of life. J Laryngol Otol. 2005; 119(7): 543-9.

3. Alt JA, Smith TL, Mace JC, Soler ZM. Sleep quality and disease severity in patients with chronic rhinosinusitis. Laryngoscope. 2013; 123(10): 2364-70.
4. Alt JA, Smith TL, Schlosser RJ, Mace JC, Soler ZM. Sleep and quality of life improvements after endoscopic sinus surgery in patients with chronic rhinosinusitis. Int Forum Allergy Rhinol. 2014; 4(9): 693-701.

5. Alt JA, Smith TL. Chronic rhinosinusitis and sleep: a contemporary review. Int Forum Allergy Rhinol. 2013; 3(11): 941-9.

6. Zwillich CW, Pickett C, Hanson FN, Weil JV. Disturbed sleep and prolonged apnea during nasal obstruction in normal men. Am Rev Respir Dis. 1981; 124(2): 158-60.

7. Suratt PM, Turner BL, Wilhoit SC. Effect of intranasal obstruction on breathing during sleep. Chest. 1986; 90(3): 324-9.

8. Storms WW. Pharmacologic approaches to daytime and nighttime symptoms of allergic rhinitis. J Allergy Clin Immunol. 2004; 114(5 Suppl): S146-53

9. Nguyen DT, Guillemin F, Arous F, Jankowski R. Assessment of quality-of-life outcomes after surgery for nasal polyposis with the DyNaChron questionnaire. Eur Arch Otorhinolaryngol. 2015; 272(2): 367-75.

10. Tosun F, Kemikli K, Yetkin S, Ozgen F, Durmaz A, Gerek M. Impact of endoscopic 
sinus surgery on sleep quality in patients with chronic nasal obstruction due to nasal polyposis. J Craniofac Surg. 2009; 20(2): 4469.

11. Kacha S, Guillemin F, Jankowski $R$ Development and validity of the DyNaChron questionnaire for chronic nasa dysfunction. Eur Arch Otorhinolaryngol. 2012; 269(1): 143-53.

12. Malm L. Assessment and staging of nasal polyposis. Acta Otolaryngol. 1997; 117(4) 465-7.

13. Jankowski R. Nasalisation in the surgical treatment of nasal polyposis. In: Önerc M, Ferguson BJ, eds. Nasal Polyposis: Pathogenesis, Medical and Surgical Treatment. Berlin, Gemany: Springer, 2010; 265-74

14. Nguyen DT, Bonfort G, Arous F, Felix-Ravelo $\mathrm{M}$, Nguyen-thi $\mathrm{PL}$, Jankowski R. Evaluation of residual symptoms: A method to assess surgical outcomes for nasal polyposis. Am J Rhinol Allergy. 2016; 30(2): e36-41.

15. Craig TJ, Ferguson BJ, Krouse JH. Sleep impairment in allergic rhinitis, rhinosinusitis, and nasal polyposis. Am J Otolaryngol. 2008; 29(3): 209-17

16. Yalamanchali S, Cipta S, Waxman J, Pott $T$, Joseph $N$, Friedman M. Effects o
Endoscopic Sinus Surgery and Nasal Surgery in Patients with Obstructive Sleep Apnea. Otolaryngol Head Neck Surg. 2014 151(1): 171-5.

17. Rubinstein I. Nasal inflammation in patients with obstructive sleep apnea. Laryngoscope. 1995; 105(2): 175-7.

18. Jiang $\mathrm{RS}$, Liang $\mathrm{KL}$, $\mathrm{H} \sin \mathrm{CH}$, Su MC. The impact of chronic rhinosinusitis on sleepdisordered breathing. Rhinology. 2016; 54(1): 75-9.

19. Ulasli SS, Gunay E, Koyuncu T, et al. Predictive value of Berlin Questionnaire and Epworth Sleepiness Scale for obstructive sleep apnea in a sleep clinic population. Clin Respir J. 2014; 8(3): 292-6.

20. Karosi T, Csomor P, Sziklai I. Tumor necrosis factor-a receptor expression correlates with mucosal changes and biofilm presence in chronic rhinosinusitis with nasal polyposis. Laryngoscope. 2012; 122(3): 504-10

21. Lennard CM, Mann EA, Sun LL, Chang AS, Bolger WE. Interleukin-1 beta, interleukin-5, interleukin-6, interleukin-8, and tumor necrosis factor-alpha in chronic sinusitis: response to systemic corticosteroids. Am J Rhinol. 2000; 14(6): 367-73.

22. Opp MR, Krueger JM. Interleukin-1 is involved in responses to sleep deprivation in the rabbit Brain Res, 1994: 639(1): 57-65.

23. Rotenberg BW, Pang KP. The impact of sinus surgery on sleep outcomes. Int Forum Allergy Rhinol. 2015; 5(4): 329-32

24. Fokkens WJ, Hellings PW. Objective measurements of nasal function: necessary before nasal surgery? Rhinology. 2014; 52(4): 289-91.

Dr Duc Trung Nguyen

Service d'Otorhinolaryngologie et

Chirurgie Cervico-Faciale

Hôpitaux de Brabois Centre Hospita-

lier Universitaire de Nancy

rue du Morvan

54511 Vandoeuvre-les-Nancy Cedex

France

Tel: +33-383-155 409

E-mail: dt.nguyen@chru-nancy.fr 